PROCEEDINGS OF THE

AMERICAN MATHEMATICAL SOCIETY

Volume 134, Number 9, September 2006, Pages 2695-2701

S 0002-9939(06)08285-2

Article electronically published on March 23, 2006

\title{
FUNCTIONS $q$-ORTHOGONAL WITH RESPECT TO THEIR OWN ZEROS
}

LUIS DANIEL ABREU

(Communicated by Carmen C. Chicone)

Abstract. In 1939, G. H. Hardy proved that, under certain conditions, the only functions satisfying

$$
\int_{0}^{1} f\left(\lambda_{m} t\right) f\left(\lambda_{n} t\right) d t=0
$$

where the $\lambda_{n}$ are the zeros of $f$, are the Bessel functions. We replace the above integral by the Jackson $q$-integral and give the $q$-analogue of Hardy's result.

\section{INTRODUCTION}

The orthogonality relations

$$
\int_{0}^{1} \sin (m \pi t) \sin (n \pi t) d t=0
$$

if $m \neq n$ and, for Bessel functions $J_{\nu}$ and their $n t h$ zero $j_{\nu n}$,

$$
\int_{0}^{1} t J_{\nu}\left(j_{\nu m} t\right) J_{\nu}\left(j_{\nu n} t\right) d t=0
$$

led J. M. Whittaker to call such functions orthogonal with respect to their own zeros [13. It is known that, under some restrictions, the only such functions are the Bessel functions. This was shown by G. H. Hardy in [4. For a remarkable big class of functions he proved that, denoting by $\lambda_{n}$ the $n t h$ zero of $f$, if $f$ satisfies

$$
\int_{0}^{1} f\left(\lambda_{m} t\right) f\left(\lambda_{n} t\right) d t=0
$$

then $f$ must be a Bessel function. The classes of functions considered by Hardy were defined in terms of the position of their zeros and their growth as entire functions, in the following terms.

Definition 1. The class $A$ is constituted by all entire functions $f$ of order less than two or of order two and minimal type of the form

$$
f(z)=z^{\nu} \prod_{n=1}^{\infty}\left(1-\frac{z^{2}}{\lambda_{n}^{2}}\right)
$$

Received by the editors October 19, 2004 and, in revised form, April 7, 2005.

2000 Mathematics Subject Classification. Primary 42C05, 33D45; Secondary 39A13.

Key words and phrases. $q$-difference equations, $q$-Bessel functions, $q$-integral.

Partial financial assistance from Centro de Matemática da Universidade de Coimbra.

(C)2006 American Mathematical Society Reverts to public domain 28 years from publication 
where $\nu>-\frac{1}{2}$. The class $B$ is constituted by all entire functions $f$ of the form

$$
f(z)=z^{\nu} F(z),
$$

where $\nu>-\frac{1}{2}$ and $F(z)$ is an entire function with real but not necessarily positive zeros, and of order one, or of order one and minimal type, with $F(0) \neq 0$.

Hardy proved that, if they satisfy (1.1), the functions on the class $A$ must be of the form $K z^{\frac{1}{2}} J_{\nu-1 / 2}(c z)$ and the functions on the class $B$ must be of the form $K J_{2 \nu}\left(c z^{1 / 2}\right)$. We will replace (1.1) with the slightly more general orthogonality relation

$$
\int_{0}^{1} f\left(\lambda_{m} t\right) f\left(\lambda_{n} t\right) d \mu(t)=0
$$

where $d \mu(t)$ is a positive defined measure in the real line and the $\lambda_{n}$ are the zeros of $f$.

This paper is organized as follows. In the second section we derive a sampling theorem for this function that was implicit in Hardy's work. Then, specializing the measure in (1.2) in order to obtain the Jackson $q$-integral, we will formulate the $q$-version of the problem, and derive the $q$-difference equations satisfied by the functions $f$. For the class $A$ we will recognize the resulting $q$-difference equation as being a parametrization of the second order $q$-difference equation derived by Meijer and Swarttouw [12 and thus prove that the only functions in class $A$ that are $q$-orthogonal with respect to their own zeros are the third Jackson $q$-Bessel functions.

\section{KRAMER KERNELS AND LAGRANGE-TYPE INTERPOLATION FORMULAS}

Suppose that $f$ satisfies (1.2). If $f \in A$, it is possible to prove that the set $\left\{f\left(\lambda_{n} t\right)\right\}$ is complete in $L^{2}[\mu,(0,1)]$ and

$$
\frac{\int_{0}^{1} f(z t) f\left(\lambda_{n} t\right) d \mu(t)}{\int_{0}^{1}\left|f\left(t \lambda_{n}\right)\right|^{2} d \mu(t)}=\frac{2 \lambda_{n}}{f^{\prime}\left(\lambda_{n}\right)} \frac{f(z)}{z^{2}-\lambda_{n}^{2}} .
$$

This was done in 4 for the case $d \mu(t)=d x$, and the proof remains the same if a general real positive measure $d \mu(t)$ is used. If $f \in B$, the set $\left\{f\left(\lambda_{n} t\right)\right\}$ is complete in $L_{q}^{2}[\mu,(0,1)]$ and

$$
\frac{\int_{0}^{1} f(z t) f\left(\lambda_{n} t\right) d \mu(t)}{\int_{0}^{1}\left|f\left(t \lambda_{n}\right)\right|^{2} d \mu(t)}=\frac{f(z)}{f^{\prime}\left(\lambda_{n}\right)\left(z-\lambda_{n}\right)} .
$$

In the next sections the measure $d \mu(t)$ will be specialized in order to obtain the $q$-integral.

The above formulas can be seen from the point of view of the Kramer sampling Lemma. The Kramer sampling Lemma [11] states that if $\left\{K\left(x, \lambda_{n}\right)\right\}$ is an orthogonal basis for $L^{2}(\mu, I)$ and for some $u \in L^{2}(\mu, I) g$ can be written in the form

$$
g(x)=\int_{I} u(t) K(t, x) d \mu(t)
$$

then $g$ admits the sampling expansion

$$
g(x)=\sum_{n=1}^{\infty} g\left(\lambda_{n}\right) S_{n}(x)
$$


where

$$
S_{n}(x)=\frac{\int_{I} K\left(t, \lambda_{n}\right) K(t, x) d \mu(t)}{\int_{I}\left|K\left(t, \lambda_{n}\right)\right|^{2} d \mu(t)} .
$$

The kernel $K(x, t)$ is called a Kramer kernel. Sometimes the integral above can be evaluated explicitly. For instance, when $K(x, t)$ is the solution of a regular Sturm-Liouville eigenvalue problem, the Kramer-type sampling expansion becomes a Lagrange-type interpolation formula with

$$
S_{n}(x)=\frac{L(x)}{L^{\prime}\left(\lambda_{n}\right)\left(x-\lambda_{n}\right)},
$$

where

$$
L(x)=\prod_{k=0}^{\infty}\left(1-\frac{x}{\lambda_{k}}\right) .
$$

As remarked by Everitt, Nasri-Roudsari and Rehberg in [2], the question of whether there exists a Lagrange interpolation formula for every Kramer kernel is open. The identities (2.1) and (2.2) provide an answer to this question when $K(x, t)=f(x t)$ (these sort of kernels are usually said to be of the Watson type) and $f$ in the classes $A$ and $B$ above. A simple application of Kramer's Lemma yields the following

Theorem 1. Let $f$ satisfy (1.2). If $f$ is in the class $A$, then every function $g$ of the form

$$
g(t)=\int_{0}^{1} u(x) f(x t) d \mu(x)
$$

has the sampling expansion

$$
g(t)=2 \sum_{n=1}^{\infty} g\left(\lambda_{n}\right) \frac{2 \lambda_{n}}{f^{\prime}\left(\lambda_{n}\right)} \frac{f(t)}{t^{2}-\lambda_{n}^{2}} .
$$

If $f$ is in the class $B$, then every function $g$ of the form (2.3) has the sampling expansion

$$
g(t)=\sum_{n=1}^{\infty} g\left(\lambda_{n}\right) \frac{f(t)}{f^{\prime}\left(\lambda_{n}\right)\left(t-\lambda_{n}\right)} .
$$

Special cases of (2.4) are known when $f$ is the Bessel [5] or the $q$-Bessel function [1]. These sampling theorems were originally obtained using special function formulae and the unitary property of the Hankel and the $q$-Hankel transform [10].

\section{Functions $q$-ORThogonal With REspect to THEIR OWN ZEROS}

3.1. Basic definitions and facts. Following the standard notations in [3, consider $0<q<1$ and define the $q$-shifted factorial for $n$ finite and different from zero as

$$
(a ; q)_{n}=(1-a)(1-a q) \ldots\left(1-a q^{n-1}\right),
$$

and the zero and infinite cases as

$$
\begin{gathered}
(a ; q)_{0}=1 \\
(a ; q)_{\infty}=\lim _{n \rightarrow \infty}(a ; q)_{n} .
\end{gathered}
$$


The $q$-difference operator $D_{q}$ is

$$
D_{q} f(x)=\frac{f(x)-f(q x)}{(1-q) x} .
$$

The $q$-analogue of the rule of the differentiation of a product is

$$
D_{q}[f(x) g(x)]=f(q x) D_{q} g(x)+g(x) D_{q} f(x),
$$

and the $q$-integral in the interval $(0, z)$ is

$$
\int_{0}^{z} f(t) d_{q} t=(1-q) \sum_{k=0}^{\infty} f\left(z q^{k}\right) z q^{k} .
$$

It is possible to define an inner product by setting

$$
\langle f, g\rangle=\int_{0}^{1} f(t) g(t) d_{q} t .
$$

The resulting Hilbert space is commonly denoted by $L_{q}^{2}(0,1)$. We will say that a function $f \in L_{q}^{2}(0,1)$ is $q$-orthogonal with respect to its own zeros in the interval $(0,1)$ if it satisfies the orthogonality relation

$$
\int_{0}^{1} f\left(\lambda_{m} t\right) f\left(\lambda_{n} t\right) d_{q} t=0,
$$

that is,

$$
\sum_{k=0}^{\infty} f\left(\lambda_{m} q^{k}\right) f\left(\lambda_{n} q^{k}\right) q^{k}=0
$$

if $n \neq m$. An example of a function satisfying such an orthogonality relation is the third Jackson $q$-Bessel function $J_{\nu}^{(3)}$ (also known in the literature as the Hahn-Exton $q$-Bessel function) defined by the power series

$$
J_{\nu}^{(3)}(x ; q)=\frac{\left(q^{\nu+1} ; q\right)_{\infty}}{(q ; q)_{\infty}} \sum_{n=0}^{\infty}(-1)^{n} \frac{q^{n(n+1) / 2}}{\left(q^{\nu+1} ; q\right)_{n}(q ; q)_{n}} x^{2 n+\nu} .
$$

Or equivalently, denoting by $j_{n \nu}(q)$ the $n$th zero of $J_{\nu}^{(3)}(x ; q)$, by the infinite product representation

$$
J_{\nu}^{(3)}(x ; q)=\frac{\left(q^{\nu+1} ; q\right)_{\infty}}{(q ; q)_{\infty}} x^{\nu} \prod_{n=1}^{\infty}\left(1-\frac{x^{2}}{j_{n \nu}^{2}(q)}\right) .
$$

The equivalence of both definitions is an easy consequence of the Hadamard factorization theorem. It is well known [9] that, if $n \neq m$,

$$
\int_{0}^{1} x J_{\nu}^{(3)}\left(q x j_{n \nu}\left(q^{2}\right) ; q^{2}\right) J_{\nu}^{(3)}\left(q x j_{m \nu}\left(q^{2}\right) ; q^{2}\right) d_{q} x=0 .
$$

This function was discussed in the context of quantum groups by Koelink [8], and the central concepts regarding its role in $q$-harmonic analysis were introduced by Koornwinder and Swarttouw [9]. The $J_{\nu}^{(k)}, k=1,2,3$, notation for the Jackson analogues of the Bessel function is due to Ismail [6], [7. 
3.2. q-difference equations. In [12, Meijer and Swarttouw proved that the general solution of the $q$-difference equation

$$
q D_{q}^{2} y(z)+\frac{1}{z} D_{q} y(z)+\left[\frac{q^{2-\nu}}{(1-q)^{2}}-\frac{\left(1-q^{\nu}\right)\left(1-q^{-\nu}\right)}{(1-q)^{2} z^{2}}\right] y(q z)=0
$$

is

$$
H_{\nu}(z)=A J_{\nu}^{(3)}\left(z ; q^{2}\right)-B Y_{\nu}\left(z ; q^{2}\right),
$$

where $Y_{\nu}\left(x ; q^{2}\right)$ is a $q$-analogue of $Y_{\nu}(x)$, the classical second solution of the Bessel differential equation. The function $Y_{\nu}\left(x ; q^{2}\right)$ is defined, if $\nu$ is not an integer, as

$$
Y_{\nu}(x ; q)=\frac{\Gamma_{q}(\nu) \Gamma_{q}(1-\nu)}{\pi}\left\{\cos (\pi \nu) q^{\nu / 2} J_{\nu}^{(3)}(x ; q)-J_{-\nu}^{(3)}\left(x q^{-\nu / 2} ; q\right)\right\}
$$

and, for $n$ an integer, as the limit

$$
Y_{n}(x ; q)=\lim _{\nu \rightarrow n} Y_{\nu}(x ; q) .
$$

It is clear that, if $\nu>0$, then $Y_{\nu}$ is unbounded near $x=0$.

Lemma 1. The general solution of the equation

$$
D_{q}^{2} y(z)+\left[\frac{M^{2} q^{\frac{3}{2}-\nu}}{\left(1-q^{2}\right)}-\frac{\left(1-q^{\nu-\frac{1}{2}}\right)\left(1-q^{-\nu-\frac{1}{2}}\right)}{\left(1-q^{2}\right) z^{2}}\right] y(q z)=0
$$

is given by

$$
f(x)=x^{\frac{1}{2}}\left\{A J_{\nu}^{(3)}\left(M x ; q^{2}\right)-B Y_{\nu}\left(M x ; q^{2}\right)\right\}
$$

Proof. Set

$$
y(x)=x^{\frac{1}{2}} H_{\nu}(M x) .
$$

Apply the operator $D_{q}$ to (3.10) and use (3.2) to obtain

$$
M D_{q} H_{\nu}(M x)=x^{-\frac{1}{2}} D_{q} y(x)+\frac{1-q^{-\frac{1}{2}}}{1-q} x^{-\frac{3}{2}} y(q x) .
$$

Now, to evaluate the second $q$-difference, again apply the operator $D_{q}$ to (3.10), but switch the role of the functions $f$ and $g$ in the formula (3.2). The result is

$$
M D_{q} H_{\nu}(M x)=q^{-\frac{1}{2}} x^{-\frac{1}{2}} D_{q} y(x)+\frac{1-q^{-\frac{1}{2}}}{1-q} x^{-\frac{3}{2}} y(x) .
$$

Applying the operator $D_{q}$ to both members gives

$$
M^{2} D_{q}^{2} H_{\nu}(M x)=q^{-1} x^{-\frac{1}{2}} D_{q}^{2} y(x)-q^{-1} x^{-\frac{3}{2}} D_{q} y(x)+\frac{\left(1-q^{-\frac{1}{2}}\right)\left(1-q^{-\frac{3}{2}}\right)}{(1-q)^{2}} x^{-\frac{5}{2}} y(q x) .
$$

Using these expressions it is not hard to see that the change of variable (3.10) transforms equation (3.8) in (3.9). This proves the lemma. 
3.3. The main results. Observe that the $q$-integral (3.3) is a Riemann-Stieltjes integral with respect to a step function having infinitely many points of increase at the points $q^{k}$, with the jump at the point $q^{k}$ being $q$. If we call this step function $\Psi_{q}(t)$, then $d \Psi_{q}(t)=d_{q} t$.

Theorem 2. If $f$ is in the class $A$ and satisfies (3.4), then $f$ must be of the form

$$
f(x)=x^{\frac{1}{2}} K J_{\nu-1 / 2}^{(3)}\left(M x ; q^{2}\right),
$$

where

$$
M^{2}=-a q^{-3}\left(1-q^{2}\right)\left(1-q^{2 \nu+1}\right)
$$

$a=-2 \sum 1 / \lambda_{n}^{2}$ and $K$ is a real constant.

Proof. Take in (2.1) $\mu(t)=\Psi_{q}(t)$ to obtain

$$
\int_{0}^{1} f(z t) f\left(\lambda_{n} t\right) d_{q} t=\frac{2 A_{n} \lambda_{n}}{f^{\prime}\left(\lambda_{n}\right)} \frac{f(z)}{z^{2}-\lambda_{n}^{2}} .
$$

With minor adaptations, the argument used in [4, page 41] can be extended to the $q$-case to deduce, from the completeness of $\left\{f\left(\lambda_{n} t\right)\right\}$ and identity (3.13), the following $q$-integral equation for $f(z)$ :

$$
a \int_{0}^{z} u^{\nu+2} f(u) d_{q} u=\left(a z^{2}+2\right) \int_{0}^{z} u^{\nu} f(u) d_{q} u-2 \frac{1-q}{1-q^{2 \nu+1}} z^{\nu+1} f(z),
$$

where $a=-2 \sum 1 / \lambda_{n}^{2}$. Then, applying the operator $D_{q}$ to both members of this equation and dividing by $z$ yields

$2 q^{\nu+1} z^{\nu} \frac{1-q}{1-q^{2 \nu+1}} D_{q} f(z)-2 q^{\nu+1} \frac{1-q^{\nu}}{1-q^{2 \nu+1}} z^{\nu-1} f(z)-a(q+1) \int_{0}^{q z} u^{\nu} f(u) d_{q} u=0$.

Using the $D_{q}$ operator again and multiplying the resulting equation by the factor $\left(1-q^{2 \nu+1}\right)(1-q)^{-1} q^{-2 \nu-1} z^{-\nu} / 2$, the result is

$$
D_{q}^{2} f(z)-\left[\frac{\left(1-q^{\nu}\right)\left(1-q^{\nu-1}\right) q^{-\nu}}{(1-q)^{2} z^{2}}+\frac{a(1+q)\left(1-q^{2 \nu+1}\right) q^{-\nu-1}}{1-q}\right] f(q z)=0 .
$$

Observe that replacing $\nu$ by $\nu-\frac{1}{2}$ and $M$ by the value given by (3.12) in (3.9) gives (3.14). Therefore, the general solution of (3.14) is

$$
f(x)=x^{\frac{1}{2}}\left\{A J_{\nu-1 / 2}^{(3)}\left(M x ; q^{2}\right)-B Y_{\nu-1 / 2}\left(M x ; q^{2}\right)\right\}
$$

with $M$ as in (3.12). But as we have seen, $Y_{\nu}$ is unbounded near $x=0$ and $f$ is analytic at $x=0$. This implies $B=0$. Therefore, (3.11) holds.

Remark 1. This agrees with orthogonality relation (3.7). To see this, just replace $\nu$ by $\nu+\frac{1}{2}$ in (3.11). The result is

$$
f(x)=A z^{\frac{1}{2}} J_{\nu}^{(3)}\left(M x ; q^{2}\right)
$$

with $M^{2}=-a q^{-3}\left(1-q^{2}\right)\left(1-q^{2 \nu+2}\right)$. To evaluate $a$, take the logarithmic derivative in (3.6) and set $x=0$. This yields

$$
\sum_{k=0}^{\infty} \frac{1}{j_{n v}^{2}\left(q^{2}\right)}=\frac{q^{2}}{\left(1-q^{2}\right)\left(1-q^{2 \nu+2}\right)} .
$$

Therefore $M=q$.

If $f \in B$ it is also possible to find the $q$-difference equation satisfied by $f$. 
Theorem 3. If $f$ is in the class $B$ and $f$ satisfies (3.4), then $f$ must satisfy the following q-difference equation:

$$
D_{q}^{2} f(z)+\frac{1}{q z} D_{q} f(z)-\left[\frac{\left(1-q^{\nu}\right)\left(1-q^{\nu-1}\right)}{\left(1-q^{2}\right) q^{\nu+1} z^{2}}-\frac{\left(1-q^{2 \nu+1}\right)(a+1)}{(1+q) q^{\nu+2} z}\right] f(q z)=0,
$$

where $a=F(0)$.

Proof. In (2.2) take $\mu(t)=\Psi_{q}(t)$ to obtain

$$
\int_{0}^{1} f(z t) f\left(\lambda_{n} t\right) d_{q} t=\frac{A_{n}}{f^{\prime}\left(\lambda_{n}\right)} \frac{f(z)}{z-\lambda_{n}} .
$$

The integral equation obtained this time is

$$
a \int_{0}^{z} u^{\nu+1} f(u) d_{q} u=(a z+2) \int_{0}^{z} u^{\nu} f(u) d_{q} u-\frac{1-q}{1-q^{2 \nu+1}} z^{\nu+1} f(z) .
$$

Use of the $q$-difference operator as in Theorem 2 establishes (3.15).

\section{REFERENCES}

[1] L. D. Abreu, A q-Sampling Theorem related to the q-Hankel transform, Proc. Amer. Math. Soc. 133 (2005), 1197-1203. MR.2117222

[2] W. N. Everitt, G. Nasri-Roudsari, J. Rehberg, A note on the analytic form of the Kramer sampling theorem, Results Math. 34 (1998), no. 3-4, 310-319. MR1652716 (99h:30036)

[3] G. Gasper and M. Rahman, Basic Hypergeometric Series, Cambridge University Press, Cambridge, UK, 1990. MR.1052153 (91d:33034)

[4] G. H. Hardy, Notes on special systems of orthogonal functions (II): On functions orthogonal with respect to their own zeros, J. Lond. Math. Soc. 14 (1939), 37-44.

[5] J. R. Higgins, An interpolation series associated with the Bessel-Hankel transform, J. Lond. Math. Soc. 5 (1972), 707-714. MR0320616 (47:9152)

[6] M. E. H. Ismail, The Zeros of Basic Bessel functions, the functions $J_{\nu+a x}(x)$, and associated orthogonal polynomials, J. Math. Anal. Appl. 86 (1982), 1-19. MR0649849 (83c:33010)

[7] M. E. H. Ismail, Some properties of Jackson's third q-Bessel function, unpublished manuscript.

[8] H. T. Koelink, The quantum group of plane motions and the Hahn-Exton q-Bessel function, Duke Math. J. 76 (1994), no. 2, 483-508. MR1302322 (96a:33023)

[9] H. T. Koelink, R. F. Swarttouw, On the zeros of the Hahn-Exton q-Bessel Function and associated q-Lommel polynomials, J. Math. Anal. Appl. 186 (1994), 690-710. MR 1293849 (95j:33050)

[10] T. H. Koornwinder, R. F. Swarttouw, On q-analogues of the Fourier and Hankel transforms, Trans. Amer. Math. Soc. 333 (1992), no. 1, 445-461. MR1069750 (92k:33013)

[11] H. P. Kramer, A generalized sampling theorem, J. Math. Phys. 38 1959/60, 68-72. MR0103786 (21:2550)

[12] R. F. Swarttouw, H. G Meijer, A q-analogue of the Wronskian and a second solution of the Hahn-Exton q-Bessel difference equation. Proc. Amer. Math. Soc. 120 (1994), no. 3, 855-864. MR.1180467 (94e:33034)

[13] J. M. Whittaker, Interpolatory function theory (1935).

Department of Mathematics, Universidade de Coimbra, Coimbra, Portugal 3001-454

E-mail address: daniel@mat.uc.pt 